\title{
Improvement of Anti-Tumor DNA Vaccination by Co-Immunization at a Distant Site with a Plasmid Encoding the Hemagglutinin-Neuraminidase Protein of Newcastle Disease Virus
}

\author{
Jing $\mathrm{Ni}^{1}$, Volker Schirrmacher ${ }^{1,2, *}$ and Philippe Fournier ${ }^{1, *}$ \\ ${ }^{1}$ German Cancer Research Center, Im Neuenheimer Feld 280, 69120 Heidelberg, Germany \\ ${ }^{2}$ IOZK, Cologne, Germany
}

\begin{abstract}
DNA vaccine encoding tumor associated antigens (TAAs) is an attractive strategy for tumor vaccine development. But its efficacy to induce efficient anti-tumor immunity needs to be improved. In this study, we combined immunization with such a plasmid at the ear pinna site (i.e.) with co-immunization with another plasmid (pHN) encoding the Hemaglutinin-Neuraminidase (HN) protein of the NDV virus at a subcutaneous site. We first tested a prophylactic immunization protocol followed by subcutaneous challenge with the ESb-lacZ lymphoma expressing the $\beta$-galactosidase protein as a surrogate tumor antigen. While i.e. vaccination with the placZ plasmid reduced tumor growth, the additional s.c. immunization with the pHN plasmid further improved this effect. We next tested a therapeutic tumor model based on the mammary carcinoma DA3-hEpCAM expressing the human EpCAM molecule. Efficient reduction of tumor growth was achieved by immunization of tumor-bearing mice with DNA plasmids encoding the human EpCAM gene only when it was combined with s.c. application of the pHN plasmid. A significantly better cross-protection against a second challenge with the parental DA3 tumor cells was only observed when mice were initially co-immunized with both plasmids.

These results demonstrate that co-immunization of a plasmid encoding the HN protein of NDV and a DNA vaccine encoding a tumor antigen significantly reduced tumor growth in mouse tumor models employing both prophylactic and therapeutic vaccination strategies. These observations point towards the HN protein of NDV as a powerful molecular adjuvant for DNA vaccines.
\end{abstract}

Keywords: DNA immunization, tumor therapy, adjuvant, TGF-ß.

\section{INTRODUCTION}

Anti-tumor DNA vaccination has been successful in eliciting tumor antigen-specific CTL responses in a certain proportion of immunized patients. However, this response is often not accompanied by a clinical response [1]. Over the last 10 years, we developed a personalized tumor vaccine which shows clinical efficacy in terms of patient's survival. This tumor vaccine, designated ATV-NDV for Autologous Tumor Vaccine modified by infection with Newcastle Disease Virus (NDV) is obtained by isolation, short term culture in vitro, irradiation and virus infection of patients' tumor cells by the lentogenic strain Ulster. Its clinical evaluation through a dozen of phase II studies with different tumor entities showed safety and clinical benefit for various cancer entities [2, for a recent review, see 3]. Significant improvement of patient survival was reported for instance for patients with breast cancer upon post-operative anti-tumor vaccination with ATV-NDV vaccine [4]. This was true also for very aggressive tumors refractory to standard treatments, such as glioblastoma and head and neck squamous cell carcinoma $[5,6]$. Recently, results of a randomized clinical study

*Address correspondence to these authors at the German Cancer Research Center (DKFZ), Im Neuenheimer Feld 280, 69120 Heidelberg, Germany; Tel: ++49 6221 29540;

E-mails: V.Schirrmacher@dkfz.de, P.fournier1@yahoo.de in colon cancer patients confirmed the effectiveness of the ATV-NDV vaccine in terms of long-term survival [7]. These results place this strategy of anti-tumor vaccination at a good ranking when compared to other randomized studies of antitumor vaccination [8].

One reason for the success of this vaccine appears to be the special properties of the avian virus NDV. It replicates selectively in tumor cells [9] and introduces into the tumor cells of the vaccine danger signals which are important for inducing/enhancing anti-tumor immune responses. Moreover, this virus shows a high safety profile since it does not replicate in normal cells due to their strong interferon response [10].

The first observation of anti-tumor effects with this type of vaccine were made in mouse tumor models and reported by us as early as 1986 [11]. Postoperative active-specific immunotherapy (ASI) with virus modified murine ESb lymphoma cells protected about $50 \%$ of mice from metastases [11]. Such protection was not obtained when the tumor vaccine was not modified by infection with NDV [11]. Protective anti-tumor immunity in the ESb lymphoma model was highly specific for the autologous tumor line [11]. Our studies performed over the last years show that the antineoplastic and immune-stimulatory properties of the ATVNDV tumor vaccine are linked to its capacity to induce interferon- $\alpha($ IFN- $\alpha)$. This key molecule is induced primarily by 
viral double-stranded RNA during NDV replication within the cytoplasm of the infected cells but also by the viral $\mathrm{HN}$ molecule $[12,13]$ which is a $74-\mathrm{kDa}$ membrane type II glycoprotein protruding from the viral envelope or from the membrane of infected cells $[14,15]$. This molecule expresses on its extracellular domain both hemagglutinin (HA) and neuraminidase (NA) activities and plays a key role in mediating the attachment of the virus to host cell receptors containing terminal sialic acid residues. Due to its agglutinating properties, the HN molecule was shown also to facilitate the contact and attachment between infected cells and other cells such as immune cells, thereby inducing co-stimulatory activities [16].

In this study, we attempted to use the viral protein $\mathrm{HN}$ as an adjuvant to enhance the anti-tumoral immune response. To this end, we constructed a plasmid DNA encoding this protein under the control of the CMV promoter $(\mathrm{pHN})$. We tested this plasmid in different mouse tumor models by combining its subcutaneous application with an immunization at the ear pinna with a DNA vaccine encoding a tumor antigen. We observed, in prophylactic and therapeutic immunization settings, that co-immunization of DNA vaccine with pHN led to the development of smaller tumors in mice. These observations show that, in anti-tumor vaccination protocols, s.c. application of DNA encoding the viral protein $\mathrm{HN}$ of NDV can be combined with intra pinna application of DNA expressing the tumor associated antigen for combating tumor growth.

\section{MATERIALS AND METHODS}

\section{Mice and Cell Lines}

Female Balb/c and DBA/2 mice were purchased from Charles River WIGA (Sulzfeld, Germany) and used at 6-8 weeks of age. Baby hamster kidney (BHK) cells (BHK-21) was cultured as described in [17]. The other cell lines ESblacZ, DA3 and DA3-hEpCAM were cultured as described in [17]. The XS106 cell line was kindly provided by Professor Takachima (University of Texas, USA) and used as a negative control for X-gal staining. Recombinant IFN- $\alpha 4$ was kindly provided by Dr Rainer Zawatzky (DKFZ, Heidelberg, Germany). X-gal staining of ESb-lacZ cells was done in 24well plate followed strategies described in [18].

\section{DNA and Immunisations}

The HN gene from NDV Ulster was amplified by PCR using the plasmid PBKTSFV-HN [12] as the template and the 2 following primers gCTCAgCCCCTTATggCCAgCTgg CAgCgTAA and gTTTAAACAACATg gACCgCgCAgTTAgCC. It was then cloned into the pTandem 1 plasmid (Novagen, Darmstadt, Germany, named here $\mathrm{pVector}$ ) to obtain the $\mathrm{pHN}$ vector (Fig. 1A). The pCMV SPORT- $\beta$-gal (betagalactosidase) vector (Invitrogen, Karlsruhe, Germany) (named here placZ) and the vector encoding the human EpCAM under the control of the CMV promoter (named phEpCAM in this study, constructed as that described in [17]) were used for immunizations. DNA immunization was performed as described in [19].

\section{Antibodies, Flow Cytometry and ELISA}

Anti-NDV-HN antibody (HN.B, see [12]) was purified by Annette Arnold (DKFZ). Anti-mouse EpCAM (G8.8) and human EpCAM (HEA125) were gifted by Dr. Gerhard Moldenhauer (DKFZ). Other antibodies are anti-H-2D ${ }^{\mathrm{d}}$ (342-12, BD Pharmingen, Heidelberg, Germany), the second antibody Goat anti-mouse IgM+G+A (SouthernBiotech, Eching, Germany) and Goat anti-rat IgG (R\&D Systems, Wiesbaden-Nordenstadt, Germany). BHK21 cells were transfected with pHN by jetPEI reagent (PolyPlus, Illkirch, France). HN expression on the cell surface was analyzed by flow cytometry with a FACSCalibur (BD Pharmingen, Heidelberg, Germany). Mouse TGF- $\beta$ was quantified using the Duo Set ELISA Development kit (R\&D Systems, Wiesbaden-Nordenstadt, Germany) according to the manufacturer's instructions.

\section{Tumor Models}

Prophylactic ESb-lacZ tumor model: DBA/2 mice were immunized twice with $50 \mu \mathrm{g} / 50 \mu \mathrm{L}$ plasmid intradermally at the ear pinna (i.e.) respecting a two-week interval. After 10 days, $2 \times 10^{5}$ ESb-lacZ cells were injected s.c. to the flank. Therapeutic DA3-hEpCAM tumor model: Balb/c mice were inoculated s.c. in the flank with $1 \times 10^{7}$ DA3-hEpCAM tumor cells. The plasmids were immunized after 7 days and then every week for 4 times. Re-challenge of DA3 cells were applied at day 59. For both models, tumor growth was followed by palpation over time after tumor inoculation.

\section{Statistics}

The statistical significance of results from experimental groups in comparison to control groups was determined by the Student's t test if not specified otherwise. All tests were two-tailed and $\mathrm{p}<0.05$ was considered to be statistically significant.

\section{RESULTS}

\section{Reduced Tumor Growth Induced by Prophylactic Co- Immunization with a Plasmid Encoding the HN Protein}

To investigate if vaccination with a DNA vaccine encoding the viral protein $\mathrm{HN}$ can improve anti-tumor DNA vaccination effects, we cloned the HN gene into a plasmid DNA under the control of the CMV promoter to obtain the plasmid $\mathrm{pHN}$ (Fig. 1A). Transfection of this plasmid into BHK21 cells induced a strong surface expression of the HN protein (Fig. 1B). We first used a prophylactic immunization protocol based on the lacZ gene coding for beta-galactosidase $(\beta-$ gal) protein as a surrogate tumor antigen. The ESb-lacZ lymphoma cell line was generated after stable transfection of a plasmid encoding the lacZ gene [19]. These cells expressed high levels of the $\beta$-gal protein as observed by X-gal staining (Fig. 2A). There was no unspecific $X$-gal staining of a lacZ negative control cell line (XS106). The DNA plasmid encoding the lacZ gene (placZ) was injected intradermally into the ear pinna (i.e.) of DBA/2 mice. To avoid interference between the different plasmids, pHN DNA was applied subcutaneously (s.c.) into to the flank of the mice as shown in Fig. (2B). The plasmids were applied 2 times by respecting a 2 week interval between each immunization. Ten days after the second immunization, the mice were challenged by s.c. application of ESb-lacZ cells. Tumor growth was followed for 20 days (afterwards some mice were euthanized when the tumor diameters reached $2 \mathrm{~cm}$ ). The tumor size and tumor weight at day 20 from individual mice are shown in Fig. 
A

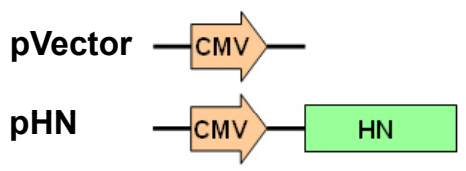

B

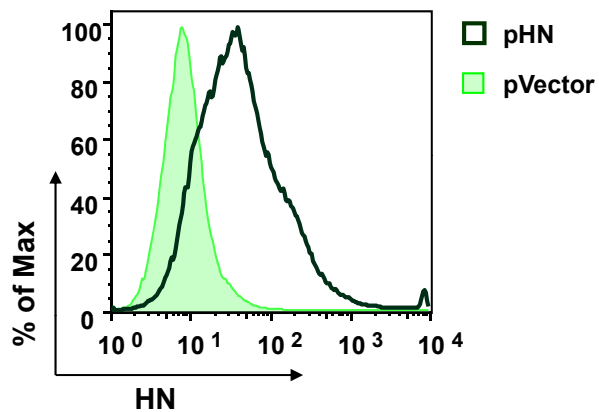

Fig. (1). Construction of a plasmid encoding the $\mathrm{HN}$ gene (pHN) and analysis of the in vitro expression of the $\mathrm{HN}$ protein.

A. Construction of the plasmid pHN. The NDV-HN gene was generated by PCR and cloned into the pTandem 1 vector to construct pCMV$\mathrm{HN}(\mathrm{pHN})$.

B. Cell surface expression of the HN protein after plasmid transfection into BHK21 cells. BHK21 cells were transfected with pHN or the control DNA (placZ). Untransfected PBS treated BHK21 cells were used as a negative control. Twenty-four hours after transfection, HN expression on the cell surface was assessed by FACS.

A

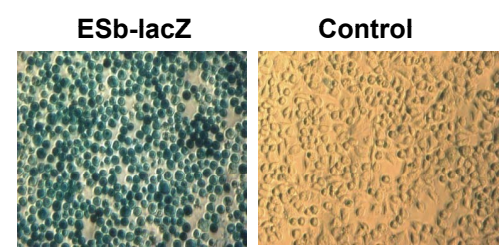

B

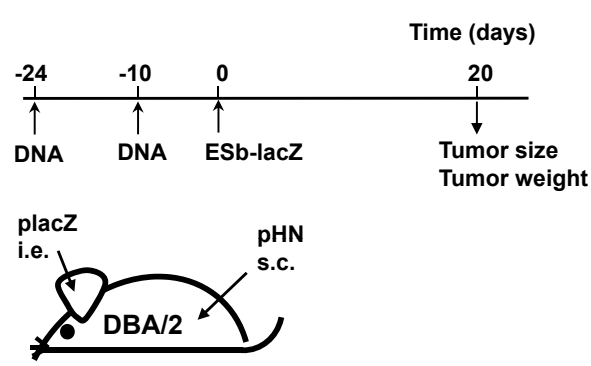

C

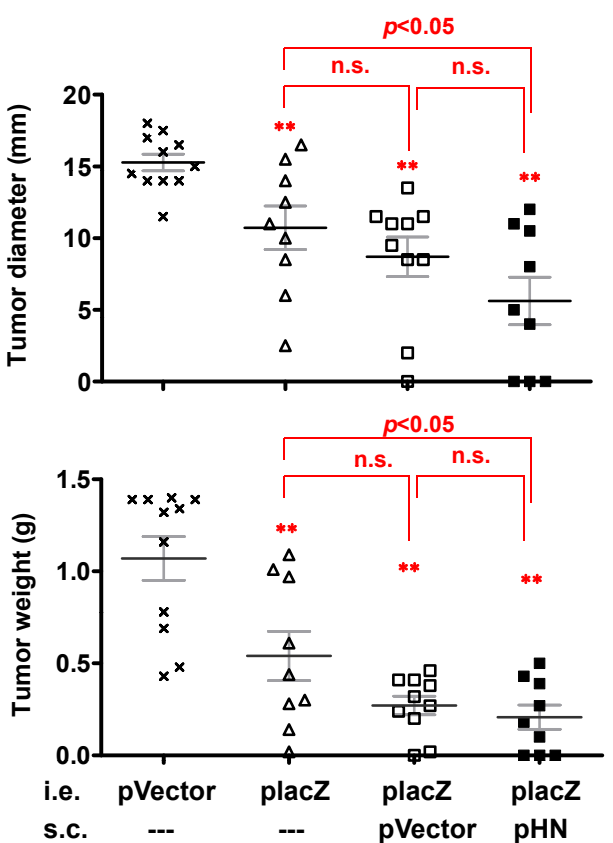

Fig. (2). Reduced tumor growth induced by co-immunization with a plasmid encoding the viral HN protein.

A. Tumor cells used in the prophylactic tumor model. We used a lymphoma cells line transduced with the lacZ gene which encodes the $\beta$ gal protein. The intracellular expression of $\beta$-gal protein was stained by X-gal staining.

B. Prophylactic vaccination protocol. DBA/2 mice $(\mathrm{n}=9 \sim 11)$ were immunized i.e. with the placZ plasmid with or without co-immunizing s.c. with the $\mathrm{pHN}$ plasmid. Mice were immunized two times with a two-week interval. Ten days after the $2^{\text {nd }}$ immunization, mice were challenged with ESb-lacZ tumor cells. Tumor growth was then followed.

C. Tumor growth. Tumor diameter in each mouse 20 days after tumor challenge is shown in the upper panel with means \pm SEM for each group. The tumor weight of each mouse for the different groups is shown in the lower panel with means \pm SEM for each group. Results of 1 representative of 3 independent experiments are shown. Similar results were obtained with 2 different experimentators.

(2C). Both parameters were significantly reduced by lacZ gene immunization compared to the control vector ( $p$ Vector) immunization. Very interestingly, co-immunization with pHN DNA induced a stronger retardation of the tumor growth, suggesting a significantly higher anti-tumor activity when compared to immunization with placZ alone. Mice coimmunized with the control vector showed no significant differences of tumor size when compared to mice immunized with the placZ plasmid alone. This shows that the reduced tumor growth observed upon $\mathrm{pHN}$ immunization is due to the HN expression and not to effects of the plasmid backbone. We conclude that co-immunization with DNA encoding the HN protein improves prophylactic DNA vaccination targeting $\beta$-gal as a surrogate tumor antigen in the ESb-lacZ tumor model. 
Reduced Tumor Growth and Reduced Systemic TGF- $\beta$ Levels by Therapeutic Co-Immunization with a Plasmid Encoding the HN Protein

We next investigated if similar effects on tumor growth could be observed when pHN was applied for therapeutic immunization. To this objective, we developed a tumor model in Balb/c mice based on the use of a syngeneic mammary carcinoma cell line DA3 and its transfectant DA3hEpCAM expressing the human EpCAM tumor antigen. Flow cytometry analysis revealed that DA3 cells show high expression of the mouse EpCAM protein on their cell surface (Fig. 3A). The stable transfectant DA3-hEpCAM cells show, in addition to the mouse EpCAM (data not shown), high expression of the human EpCAM molecule (Fig. 3A). The DA3 tumor cell line expresses low levels of MHC class I molecules (here we stained H2-D ${ }^{\mathrm{d}}$ ) while the DA3-hEpCAM cell line expresses these molecules to a slightly higher level. The expression of $\mathrm{H} 2-\mathrm{D}^{\mathrm{d}}$ molecules by both cell lines could become up-regulated by IFN- $\alpha$ treatment in a dosedependent way (Fig. 3B). The mean staining intensity was increased 30 times and 17 times, respectively for the DA3 and DA3-hEpCAM cell lines when treated with 1000 $\mathrm{IU} / \mathrm{mL}$ IFN- $\alpha$ (as shown in Fig. (3C)). Mice injected s.c. with DA3-hEpCAM cells developed tumors of 8-10 mm diameter within 1 week. Therapeutic vaccinations were then started and repeated 4 times at weekly intervals (Fig. 4A). As before, the plasmid encoding hEpCAM was given i.e. whereas the pHN vector was injected s.c. (Fig. 4A). Tumor growth retardation could be observed from day 21 onwards (data not shown). Tumor sizes at day 28 are shown in Fig. (4B). Vaccination with phEpCAM alone had no significant effect on the tumor inhibition. Co-immunization with the $p$ Vector control plasmid caused a significant $(p=0,02)$ reduction of tumor size. But co-application of $\mathrm{pHN}$ led to a further significant $(\mathrm{p}=0,002)$ tumor reduction compared to the $\mathrm{pVec}$ tor control (Fig. 4B). In addition, the levels of TGF- $\beta$ in the sera of the mice treated by application of both plasmids phEpCAM (i.e.) and pHN (s.c.) were significantly lower than in mice treated only with pVector (i.e.) (Fig. 4C). We conclude that co-injection of the plasmid pHN improves anti-tumor effects of a therapeutic DNA vaccine in the DA3hEpCAM tumor model.

\section{Reduced Tumor Growth Upon Re-Challenge of Co- Immunized Animals with Parental DA3 Tumor Cells}

Immunized mice from the experiment in Fig. (4) were rechallenged at day 59 at the contralateral flank with the parental DA3 tumor cells (Fig. 5A) which express the murine
A

DA3

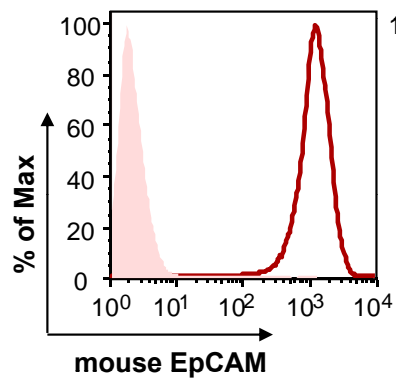

B

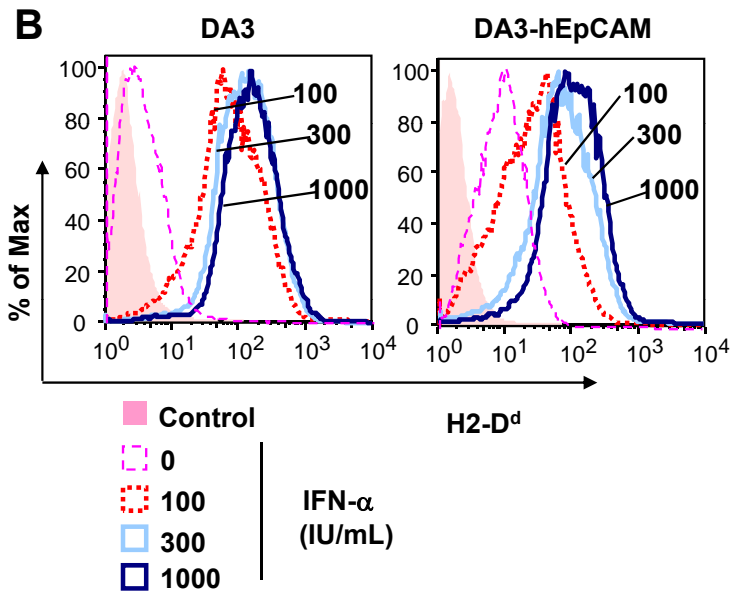

DA3-hEpCAM

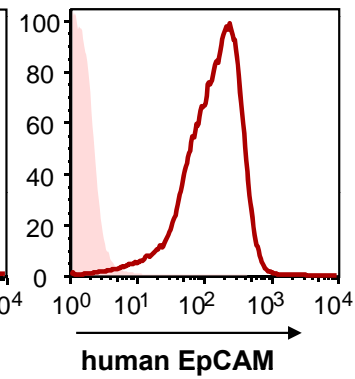

C

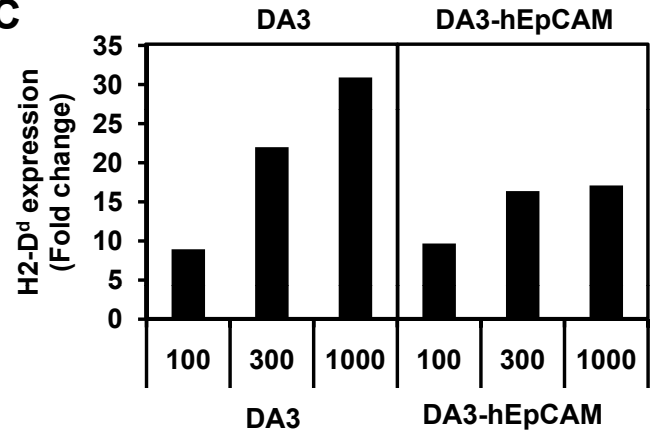

IFN- $\alpha(I U / m L)$
Control

Anti-EpCAM

Fig. (3). Characterization of DA3 and DA3-hEpCAM cells.

A. TAA expression on the DA3 and DA3-hEpCAM cells. Cell surface expression of the mouse EpCAM protein on DA3 tumor cells and the human hEpCAM protein on DA3-hEpCAM cells was analyzed by flow cytometry. Control was stained by the second antibody.

B. IFN- $\alpha$ improved MHC class I expression on DA3 and DA3-EpCAM cells. DA3 and DA3-hEpCAM was cultured in the presence of different amount of IFN- $\alpha(0,100,3000,1000 \mathrm{IU} / \mathrm{ml})$ for 3 days. Levels of MHC class I expression was followed by FACS after staining with a H2- $\mathrm{D}^{\mathrm{d}}$ specific antibody. Control was stained by an isotype matched antibody of different specificity.

C. Fold increase of H2-D ${ }^{d}$ expression after IFN- $\alpha$ treatment. The relative fold increase of H2-D ${ }^{d}$ expression on the two cell lines after IFN- $\alpha$ treatment was calculated by comparing their mean fluorescence levels to the original expression without IFN- $\alpha$ treatment. 
A

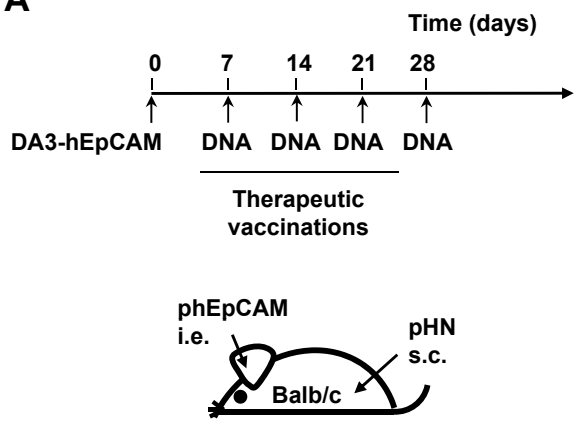

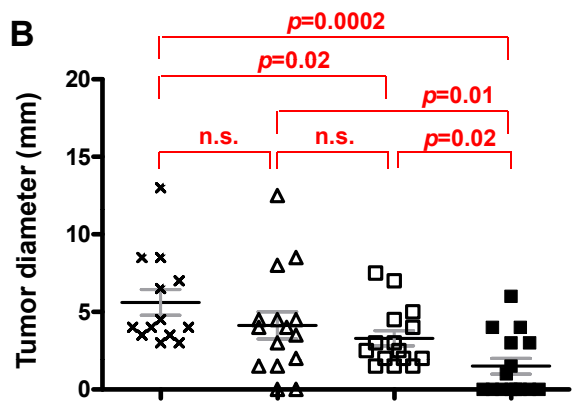

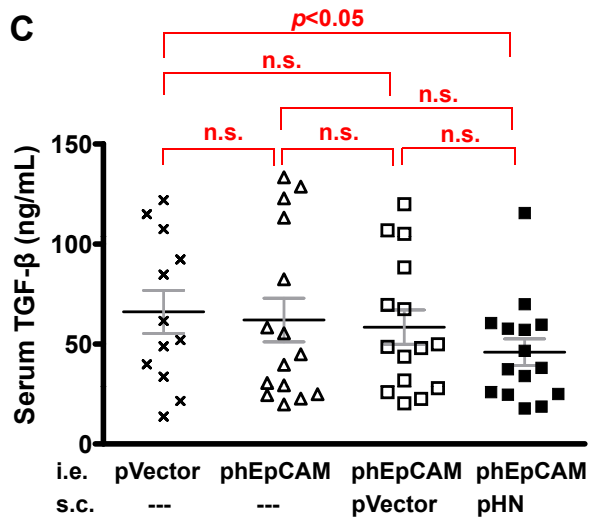

Fig. (4). Reduced tumor growth and systemic TGF- $\beta$ levels by therapeutic co-immunization with a plasmid encoding the HN protein. A. Therapeutic vaccination protocol. At day $0,1 \times 10^{7}$ DA3-hEpCAM cells were applied s.c. to Balb/c mice $(\mathrm{n}=10 \sim 15)$. Seven days later, mice were treated 4 times respecting 1 week interval between each immunization consisting in i.e. application of phEpCAM and s.c. coimmunization of $\mathrm{pHN}$ or $\mathrm{p}$ Vector. Tumor growth was then followed over time.

B. Decreased tumor growth upon pHN co-vaccination. The tumor diameter at day 28 from individual mice is shown with means \pm SEM for each group. Similar results were obtained with 2 different experiments.

C. Decreased levels of serum TGF- $\beta$ upon $\mathbf{p H N}$ co-immunization. The sera were taken at day 49 after the tumor inoculation and measured by ELISA for TGF- $\beta$ level. Results are shown as the level from individual mice with means \pm SEM.

EpCAM. Only the mice which had been treated by coimmunization with phEpCAM and pHN showed a highly significant reduction of the tumor grow th two weeks after the re-challenge. No significant tumor retardation was seen in the groups vaccinated with phEpCAM alone or coimmunized with pVector (Fig. 5B). These data suggest that co-immunization with $\mathrm{pHN}$ and phEpCAM not only induces the strongest therapeutic effects against the tumor expressing the xenogeneic target hEpCAM but also causes crossprotection against the parental tumor expressing the murine EpCAM as a self-antigen.

Taken together, our results show that co-administration of the pHN plasmid by the s.c. route augments the anti-tumor effects of anti-tumor DNA vaccines injected into the ear pinna.

\section{DISCUSSION}

In the late nineteenth century, Dr. William Coley discovered that cancer patients suffering from bacterial infection showed reduced tumor burden. This observation suggested that immune activation against a pathogen may be beneficial in cancer treatment [20]. Since then, the use of live attenuated and heat killed microorganisms has been explored to generate more efficient anti-tumor immunotherapy. These immuno-enhancers improve specific adaptive immunity mainly through stimulating or modulating the innate immune system. Some strategies have shown beneficial effects for certain cancer entities as for example bladder cancer with
Bacille Calmette Guérin (BCG) [21], although the precise mechanism is unclear. Here we studied the effect on tumor growth in vivo of co-immunization with $\mathrm{pHN}$, a plasmid encoding the well-defined HN protein of NDV, in addition to vaccination with a plasmid encoding a tumor antigen. We observed in mouse models that subcutaneous immunization with pHN improved the effectiveness of a DNA tumor vaccine applied at ear pinna site.

Genetic immunization is a promising approach for effective immunization against a range of antigens. By exploiting the transcription and translation mechanisms of the host, foreign DNA can lead to the endogenous synthesis of one or more antigens that results in the induction of humoral and cellular immune responses [22]. This approach of using DNA plasmids is expected to be applicable also for tumor prevention or therapy. However, due to the low immunogenicity of most tumor antigens and because of the presence of tumor-derived immune suppressive factors in tumor patients, the anti-tumor immune responses induced by DNA vaccination need to be improved, for instance by combining them with immuno-stimulating molecules. $\mathrm{HN}$ in this study is used as such a molecule for anti-tumor DNA vaccines. We showed the promising improvement for both prophylactic and therapeutic vaccinations by co-immunization of $\mathrm{HN}$ encoding DNA to a different site (s.c. to the flank) to where the TAA encoding DNA was injected (i.e.). 
A

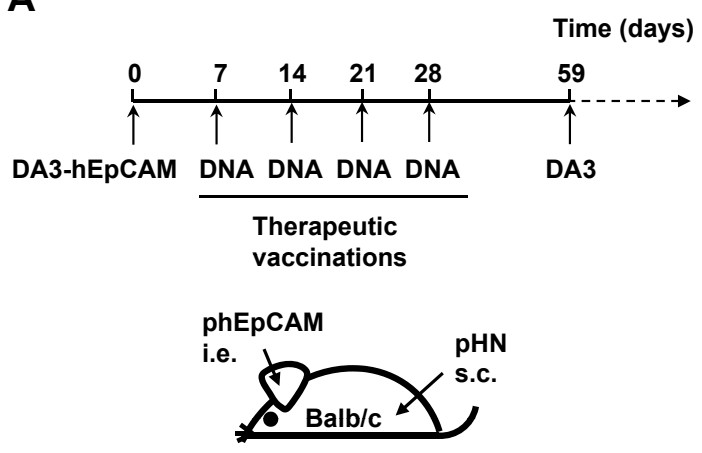

B

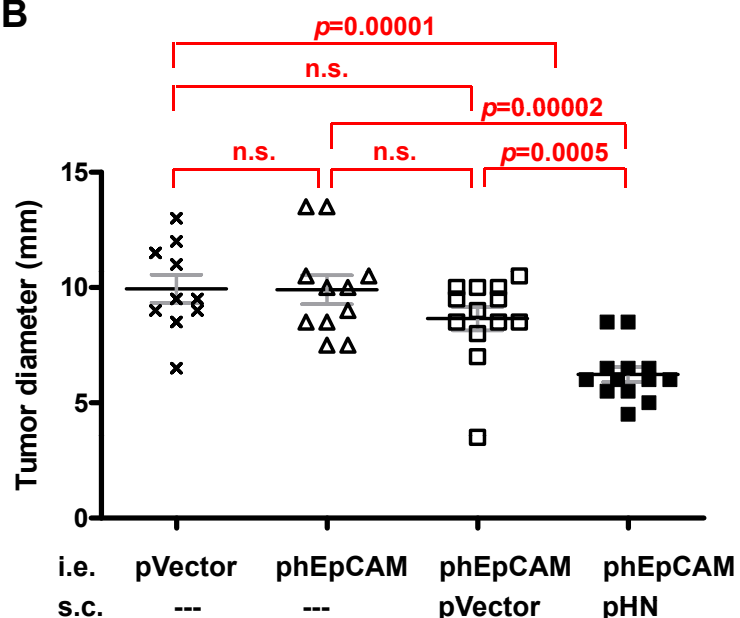

Fig. (5). Reduced tumor growth of the re-challenged tumor after therapeutic co-immunization with a plasmid encoding the HN protein.

A. Tumor re-challenge protocol. Mice were inoculated with DA3-hEpCAM tumor cells and treated four times with DNA vaccines (as indicated in Fig. 4). Fifty-nine days later, the mice were re-challenged at the contralateral flank by s.c. application of $1 \times 10^{7}$ DA3 tumor cells. Growth of the DA3 tumor was then followed over the time.

B. Decreased DA3 tumor growth upon pHN co-vaccination. The DA3 tumor diameter after 14 days of the rechallenge from individual mice is shown with means \pm SEM for each group. Similar results were obtained with 2 different experiments.

In this study, the DNA anti-tumor vaccine was applied to the ear pinna of the mice. This site has been shown previously to be superior to muscle or flank skin for the induction of a strong $\mathrm{T}$ cell mediated immune response [23]. The ear pinna microenvironment was found to exert a Th1 polarization effect [24].

Several studies reported that the immune responses induced by DNA vaccines could be improved significantly by the co-administration of cytokine-encoding plasmids [25, 26]. Nevertheless, novel adjuvants are still to be investigated in order to optimize DNA anti-tumor vaccination. We demonstrate here the efficiency of $\mathrm{HN}$-encoding DNA in augmenting the efficiency of a DNA anti-tumor vaccine. The $\mathrm{HN}$ molecule has been shown to stimulate a strong natural IFN- $\alpha$ response $[12,13]$ and it also can provide T cell costimulatory function $[16,27]$. In addition, we reported recently that $\mathrm{HN}$ serves as a ligand for NKp44 and NKp46 and activates NK cells to produce interferon- $\gamma$, TNF- $\alpha$ and to become cytotoxic [28].

IFN- $\alpha$ belongs to type I IFNs and exhibits strong antiviral activity [29]. It can also activate a number of indirect antiviral processes, including natural killer cell activation [30], up-regulation of antigen cross-presentation [31] and increased antibody production [32]. In addition, IFN- $\alpha$ is capable of positively or negatively affecting the generation, terminal differentiation, and function of various DC populations [33, 34]. It mediates the cross-priming of CD8+ T cells by antigen-presenting cells [35], promotes or blunts type 1 Th cell responses [36], and stimulates the clonal proliferation and longevity of activated or memory $\mathrm{T}$ cells [37]. Therefore, this molecule can be used as an immuno-modulator to enhance the immunogenicity of DNA vaccine. In breast cancer patients, intratumoral levels of IFN- $\alpha$ have been correlated to better prognosis [38]. Since NDV-HN protein can induce IFN- $\alpha$ production, this could be one of the reasons for the reduced tumor growth observed in our tumor models upon immunization with pHN. In addition, we observed that IFN- $\alpha$ could up-regulate the expression of MHC class I on the DA3 and DA3-hEpCAM tumor cells, thereby making them more sensitive to $\mathrm{T}$ cell-mediated cytotoxicity. This might help $\mathrm{T}$ cell mediated anti-tumor responses.

HN gene co-immunization was also observed in this study to lead to decreased peripheral TGF- $\beta$ levels in tumorbearing mice under therapeutic vaccination. This is important since tumors are known to be able to create a tolerogenic environment, notably through secretion of suppressive cytokines such as TGF- $\beta$ and IL-10 and through enhancing suppressive cell activity including MDSCs, tolerogenic DCs, tumor-associated macrophages and Tregs [39-41]. Therefore, a lower level of TGF- $\beta$ by pHN immunization mice could also be considered to be correlated with better prognosis.

In conclusion, to understand the inefficiency of DNA vaccines in humans, it is necessary to determine the pathways to generate appropriate signals for immune induction. We described here the use of a DNA sequence encoding the $\mathrm{HN}$ protein of NDV as a potent tool to augment the effectivity of anti-tumor DNA vaccines.

\section{ACKNOWLEDGEMENTS}

This study was also supported by a grant from the European Commission during the research program FP6 called MOLEDA (acronym standing for Molecular Optimization of Laser / Electrotransfer DNA Administration into muscle and skin for gene therapy). We thank the DAAD (German Academic Exchange Service) who supported Jing Ni during her $\mathrm{PhD}$ thesis. This work was also supported by grants from the Monsi Foundation, Monaco. The writing of this manuscript would not been possible without the financial support of the Dr. Kleist Stiftung (Berlin, Germany) and the ChristianBerndt Stiftung (Rostock, Germany). 


\section{REFERENCES}

[1] Slovin SF. Tribulations or triumphs in prostate cancer immunotherapy: on the road to victory? Expert Rev Anticancer Ther 2008; $8(3): 465-74$.

[2] Schirrmacher V. Clinical trials of anti-tumor vaccination with an autologous tumor cell vaccine modified by virus infection: improvement of patient survival based on improved anti-tumor immune memory. Cancer Immunol Immunother 2005; 54(6): 587-98.

[3] Schirrmacher V, Fournier P. Newcastle disease virus: a promising vector for viral therapy, immune therapy, and gene therapy of cancer. Methods Mol Biol 2009; 542: 565-605.

[4] Ahlert T, Sauerbrei W, Bastert G, et al. Tumor cell number and viability as quality and efficacy parameters of autologous virus modified cancer vaccines. J Clin Oncol 1997; 15: 1354-66.

[5] Steiner HH, Bonsanto MM, Beckhove P, et al. Anti-tumor vaccination of patients with glioblastoma multiforme: a pilot study to assess: Feasibility, safety and clinical benefit. J Clin Oncol 2004; 22 (21): 4272-81.

[6] Karcher J, Dyckhoff G, Beckhove P, et al. Anti-tumor vaccination with HNSCC with autologous virus-modified tumor cells. Cancer Res 2004; 64 (21): 8057-61.

[7] Schulze T, Kemmner W, Weitz J, Wernecke KD, Schirrmacher V, Schlag PM. Efficiency of adjuvant active specific immunization with Newcastle disease virus modified tumor cells in colorectal cancer patients following resection of liver metastases: results of a prospective randomized trial. Cancer Immunol Immunother 2009; 58(1): 61-9.

[8] Fournier P, Schirrmacher V. Randomized clinical studies of antitumor vaccination: state of the art in 2008. Expert Rev Vaccines 2009; 8(1): 51-66.

[9] Fiola C, Peeters B, Fournier P, Arnold A, Bucur M, Schirrmacher V. Tumor-selective replication of Newcastle Disease Virus: association with defects of tumor cells defence. Int J Cancer 2006; 15; 119 (2): 328-38

[10] Wilden H, Fournier P, Zawatzky R, Schirrmacher V. Expression of RIG-I, IRF3, IFN-beta and IRF7 determines resistance or susceptibility of cells to infection by Newcastle Disease Virus. Int J Oncol 2009; 34(4): 971-82.

[11] Heicappell R, Schirrmacher V, von Hoegen P, Ahlert T, Appelhans B. Prevention of metastatic spread by postoperative immunotherapy with virally modified autologous tumor cells. I: parameters for optimal therapeutic effects. Int J Cancer 1986; 37: 569-77.

[12] Zeng J, Fournier P, Schirrmacher V. Induction of interferon-alpha and tumor necrosis factor-related apoptosis-inducing ligand in human blood mononuclear cells by hemagglutinin-neuraminidase but not F protein of Newcastle disease virus. Virology 2002; 297: 1930 .

[13] Zeng J, Fournier P, Schirrmacher V. Stimulation of human natural interferon-alpha response via paramyxovirus hemagglutinin lectincell interaction. J Mol Med 2002; 80: 443-51.

[14] Fournier P, Zeng J, Schirrmacher V. Two ways to induce innate immune responses in human PBMCs: paracrine stimulation of IFNalpha responses by viral protein or dsRNA. Int J Oncol 2003; 23 : 673-80.

[15] Fournier P, Zeng J, Von Der Lieth CW, Washburn B, Ahlert T, Schirrmacher V. Importance of serine 200 for functional activities of the hemagglutinin-neuraminidase protein of Newcastle Disease Virus. Int J Oncol 2004; 24: 623-34.

[16] Ertel C, Millar NS, Emmerson PT, Schirrmacher V, von Hoegen P. Viral hemagglutinin augments peptide specific cytotoxic T-cell responses. Eur J Immunol 1993; 23: 2592-96.

[17] Ni J, Nolte B, Arnold A, Fournier P, Schirrmacher V. Targeting anti-tumor DNA vaccines to dendritic cells via a short CD11c promoter sequence. Vaccine 2009; 27(40): 5480-7.

[18] Krüger A, Umansky V, Rocha M, Hacker HJ, Schirrmacher V, von Hoegen P. Pattern and load of spontaneous liver metastasis dependent on host immune status studied with a LacZ transduced lymphoma. Blood 1994; 84(9): 3166-74.
Förg P, von Hoegen P, Dalemans W, Schirrmacher V. Superiority of the ear pinna over muscle tissue as site for DNA vaccination. Gene Ther 1998; 5(6): 789-97.

[20] Hoption CSA, van Netten JP, van Netten C. Dr. William Coley and tumour regression: a place in history or in the future. Postgrad Med J 2003; 79: 672-80.

[21] Oosterlinck W. Guidelines on diagnosis and treatment of superficial bladder cancer. Minerva Urol Nefrol 2004; 56(1): 65-72.

[22] Cohen AD, Boyer JD, Paul DB. Modulating the immune response to genetic immunization. FASEB J 1998; 12: 1611-26.

[23] Forg P, von Hoegen P, Dalemans W, Schirrmacher V. Superiority of the ear pinna over muscle tissue as site for DNA vaccination. Gene Ther 1998; 5: 789-97.

[24] Jurianz K, von Hoegen P, Schirrmacher V. Superiority of the ear pinna over a subcutaneous tumour inoculation site for induction of a Th1-type cytokine response. Cancer Immunol Immunother 1998; 45(6): 327-33

[25] Kim JJ, Simbiri KA, Sin JI, et al. Cytokine molecular adjuvants modulate immune responses induced by DNA vaccine constructs for HIV-1 and SIV. J Interferon Cytokine Res 1999; 19: 77-84.

[26] Min W, Lillehoj HS, Burnside J, Weining KC, Staeheli P, Zhu JJ. Adjuvant effects of IL-1, IL-2, IL-8, IL-15, IFN-. IFN- TGF- 4 and lymphotactin on DNA vaccination against Eimeria acervulina. Vaccine 2002; 20: 267-74.

[27] Haas C, Ertel C, Gerhards R, Schirrmacher V. Introduction of adhesive and costimulatory immune functions into tumor cells by infection with Newcastle Disease Virus. Int J Oncol 1998; 13: 1105-15.

[28] Jarahian M, Watzl C, Fournier P, et al. Activation of natural killer cells by newcastle disease virus hemagglutinin-neuraminidase. J Virol 2009; 83(16): 8108-21.

[29] Katze MG, He Y, Gale M Jr. Viruses and interferon: a fight for supremacy. Nat Rev Immunol 2002; 2(9): 675-87.

[30] Biron CA, Nguyen KB, Pien GC, Cousens LP, Salazar-Mather TP Natural killer cells in antiviral defense: function and regulation by innate cytokines. Annu Rev Immunol 1999; 17: 189-220.

[31] Le Bon A, Durand V, Kamphuis E, et al. Direct stimulation of T cells by type I IFN enhances the CD8+ T cell response during cross-priming. J Immunol 2006; 176(8): 4682-9.

[32] Gavin AL, Hoebe K, Duong B, et al. Adjuvant-enhanced antibody responses in the absence of toll-like receptor signaling. Science 2006; 314(5807): 1936-8.

[33] Belardelli F. Role of interferons and other cytokines in the regulation of the immune response. APMIS 1995; 103: 161-79.

[34] Gabriele L, Borghi P, Rozera C, et al. IFN- $\alpha$ promotes the rapid differentiation of monocytes from patients with chronic myeloid leukemia into activated dendritic cells tuned to undergo full maturation after LPS treatment. Blood 2004; 103: 980-87.

[35] Le Bon A, Etchart N, Rossmann C, et al. Cross-priming of CD8+ T cells stimulated by virus-induced type I interferon. Nat Immunol 2003; 4: 1009-15.

[36] Farrar JD, Murphy KM. Type I interferons and T helper development. Immunol Today 2000; 21: 484-89.

[37] Bogdan C. The function of type I interferons in antimicrobial immunity. Curr Opin Immunol 2000; 12: 419-24.

[38] Domschke C, Schuetz F, Ge Y, et al. Intratumoral cytokines and tumor cell biology determine spontaneous breast cancer-specific immune responses and their correlation to prognosis. Cancer Res 2009; 69(21): 8420-8.

[39] Kim R, Emi M, Tanabe K. Cancer cell immune escape and tumor progression by exploitation of anti-inflammatory and pro-inflammatory responses. Cancer Biol Ther 2005; 4: 924-33.

[40] Gajewski TF, Meng Y, Harlin H. Immune suppression in the tumor microenvironment. J Immunother 2006; 29: 233-40.

[41] Movahedi K, Guilliams M, Van den Bossche J, et al. Identification of discrete tumor-induced myeloid-derived suppressor cell subpopulations with distinct $\mathrm{T}$ cell-suppressive activity. Blood 2008; 111: 4233-44. 